\title{
Investigating the Relationship Between Water Production and Interfacial Activity of $\Gamma$-oryzanol, Ethyl Ferulate, and Ferulic Acid During the Peroxidation of Bulk Oil
}

Mohamad Reza Toorani

Shiraz University

Mohamad-Taghi Golmakani ( $\sim$ golmakani@shirazu.ac.ir)

Shiraz University

\section{Research Article}

Keywords: Critical micelle concentration, Interfacial phenomena, Oxidation kinetic, Sigmoidal equation

Posted Date: May 13th, 2021

DOI: https://doi.org/10.21203/rs.3.rs-509183/v1

License: (c) (i) This work is licensed under a Creative Commons Attribution 4.0 International License.

Read Full License 
1 Investigating the relationship between water production and interfacial

2

3

5

6

7

8

$9 \quad *$ Corresponding author

10 E-mail: golmakani@shirazu.ac.ir

11 Tel: (+98) 71-36138243 Fax: (+98) 71-32286110 71441-65186, Shiraz, Iran. peroxidation of bulk oil

\author{
Mohamad Reza Toorani, Mohamad-Taghi Golmakani*
}

Department of Food Science and Technology, School of Agriculture, Shiraz University, Postal Code activity of $\gamma$-oryzanol, ethyl ferulate, and ferulic acid during the 


\section{Abstract}

13 In this study, lecithin (as a surfactant) was added to promote the inhibitory-mechanism of $\gamma$ oryzanol, ethyl-ferulate and ferulic acid (based on the interfacial phenomena) so as to inhibit the oxidation of stripped sunflower oil. Monitoring the amount of water production as a byproduct of oxidation showed that the water content of the lipid system increased remarkably

17 through the oxidation progress. Lecithin enhanced the critical concentration of hydroperoxides in reverse micelles, compared to the basic state (14.8 vs. $9.2 \mathrm{mM})$, thereby improving the hydrogen-donating mechanism of antioxidants. The size of reverse micelles increased progressively during the oxidation, while two breakpoints were pointed out in the micelles growth, i.e. at the end of the initiation and the propagation phases. Based on the kinetic data, ferulic acid showed the highest antioxidant activity (23.4), compared to ethyl-ferulate (15.5) and $\gamma$-oryzanol (13.7). Generally, lecithin enhanced antioxidant activity ( $65 \%)$ by improving the interfacial performance of antioxidants.

Keywords: Critical micelle concentration, Interfacial phenomena, Oxidation kinetic, 
Oxidation reaction is one of the main concerns in reducing the quality and deterioration of vegetable oils. The process of oxidation occurs more rapidly in oils with polyunsaturated fatty acids and comprises three consecutive periods (i.e. initiation, propagation, and termination phases). The process involves the production of hydroperoxides (ROOHs) which is of particular importance as precursors of all oxidation products. ${ }^{1}$ Monitoring the accumulation of ROOHs during different stages of oxidation can provide valuable information for researchers about events during different stages and transfer of phases. Undoubtedly, such an information can contribute to the inhibition of oxidative reactions. reaction. ${ }^{2}$ This process continues until the point where suddenly the slope of ROOHs production increases dramatically. Known as the $\mathrm{ROOH}_{I P}$, the said point coincides with the phase transition from the initiation phase to the propagation phase. ${ }^{3}$ By passing this stage, the slope of ROOHs production continues to increase until it reaches its highest level in the middle of the propagation phase. From this point onwards, known as the turning point or the maximum rate $\left(\mathrm{M}_{\mathrm{R}}\right)$, the decomposition reaction of ROOHs begins. ${ }^{4}$ The occurrence of this reaction as an equilibrium reaction continues until it reaches a balance between production and decomposition of ROOHs. ${ }^{5}$ This irreversible point is considered as $\mathrm{ROOH}_{\max }$ (maximum achievable concentration) and is associated with surpassing the decomposition rate of ROOHs, compared to their formation rate. ${ }^{1,4}$ Such behavior has good potential to be interpreted by a sigmoidal kinetic model in which several important indices exist. One of these indices is the rate constant of pseudo-first-order $\left(k_{\mathrm{f}}\right)$, known as a measure of the formation of ROOHs (or oxidizability of lipid systems) in the propagation phase. Another criterion is the rate constant 
phase. ${ }^{5,6}$ Valuable information can result from combining the indices of the sigmoidal model and by generalizing them to physicochemical events that occur at the oxidation phase transfer. Ferulic acid (FRA) is a well-known antioxidant in many products of herbal origin. In the benzene ring of this hydroxycinnamic acid, methoxy and hydroxyl groups occur simultaneously and adjacent to each other. The methoxy group in the benzene ring can make an intramolecular hydrogen bond by creating a hydrogen bridge with its nearby OH-group. ${ }^{7}$ This reaction often occurs in nonpolar environments, causing the hydrogen donating mechanism (by hydroxyl group) to become somewhat inactive. ${ }^{8}$ However, after hydrogen separation, the presence of the methoxy group can stabilize the remaining electrons, as this happens by their delocalization, and facilities the mechanism of electron transfer. ${ }^{9}$ This paradoxical behavior of the methoxy group causes performative changes in antioxidant mechanisms according to their functional environment. Phytosteryl ferulate or gamma oryzanol (GOR) is a renown natural antioxidant that originates from rice bran oil. ${ }^{10}$ This antioxidant has higher solubility due to its bulky structure, compared to ferulic acid in the lipid substrate. Ethyl ferulate (EFR) is also derived from ferulic acid with more lipophilic characteristics and less antioxidant capacity. Naturally, it is isolated from giant fennel. ${ }^{11}$

Vegetable oils contain small amounts of water, although they originate from oil seeds and through refining processes and surfactants that may exist naturally in the source (such as mono- or di-acylglycerols and phospholipids) or which could be produced during the oxidation process (e.g. ROOHs, alcohols, aldehydes, and ketones). In the presence of water, these surface-active agents can create reverse micelles or lamellar structures by reducing interfacial tension. Thus, vegetable oils contain regular physical structures and, as a matter of fact, oxidation reactions occur in these microreactors. ${ }^{12,13}$ Molecules with higher polarity, compared to the polarity of triacylglycerols (such as antioxidants or free radicals), exhibit a greater tendency to migrate to water-oil interfaces where inhibitory reactions have a high chance of 
happening. ${ }^{14,15}$ As oxidation progresses further and adds to the production of ROOHs, the number of micelles and their size increase until they reach a critical micelles concentration (CMC), followed by an eventual collapse. This point is exactly equal to $\mathrm{ROOH}_{\mathrm{IP}}$ wherein the oxidation process enters the propagation phase by releasing a large volume of ROOHs throughout the environment and optimizes collisions between free radicals. ${ }^{16}$

Lecithin (LEC) as a phospholipid is an amphiphilic compound that can protect vegetable oils against oxidation. In a relevant literature review, various roles of performance have been suggested for this compound in vegetable oils and in preventing their oxidation. Several of these performative roles include the regeneration of primary antioxidants, metal chelating and the establishment of an oxygen barrier between the oil and air interfaces. ${ }^{15,17-19}$ Another important performance of LEC can be seen in relation to its role in supporting the formation of reverse micelles during the oxidation process. Considering the fact that phospholipids can markedly reduce interfacial tension, the number and size of microreactors are likely to increase significantly in the presence of specialized surfactants. As a result, there can be an increase in the acceptance capacity of ROOHs in these structures. Since, relatively polar antioxidants are precisely located in the interfaces of micro-micelles, ${ }^{13}$ more interactions can occur between antioxidant molecules and ROOHs. This physical role of LEC can lead to a delay in achieving CMC and to an increase in the duration of the induction period (IP). However, less attention has been given to a part of the antioxidant that comes in the contact area between oil/storage-container and air/oil (due to the difference in the polarity). ${ }^{20}$ The presence of surface-active agents is assumed to excite the movement of this part of antioxidant molecules into the water-oil interface by increasing the number of microreactors.

In this regard, the present study aimed to investigate the antioxidant activity of RFA and its derivatives with different alkyl chains (EFR and GOR) in the presence of LEC to elucidate the effects of interfacial phenomena on bulk oil peroxidation. Furthermore, various 
oxidation indices pertained to the initiation and the propagation phases were evaluated to clarify the details of physicochemical events that occurred during the oxidation process.

\section{Materials and Methods}

\section{Materials}

Refined sunflower oil was purchased from Golbarg-e-Baharan Company (Karaj) as an oxidative substrate. The GOR (CAS No. 11042-64-1) was purchased from TCI Chemicals Company (Tokyo, Japan). Meanwhile, FRA (CAS No. 1135-24-6), EFR (CAS No. 4046-020), and LEC (CAS No. 8002-43-5) were purchased from Sigma Aldrich (St. Louis, MO). All other solvents, chemicals and standard markers were purchased from Merck (Darmstadt, Germany) and Sigma Aldrich companies.

\section{Oil purification process}

The bulk oils contained minor components that may interfere with the performance of antioxidants or may affect the oxidation process. To eliminate these components, the purification process was performed by an adsorption chromatography column. To this end, two-glass column series were used (columns size, $36 \mathrm{~cm}$ height and $29 \mathrm{~mm}$ internal diameter). Each column comprised three layers of adsorbent (from the top layer to the bottom; $5 \mathrm{~g}$ of activated carbon, $30 \mathrm{~g}$ of silica gel, and $50 \mathrm{~g}$ of aluminum oxide 60 ). All sorbents were activated at $180{ }^{\circ} \mathrm{C}$ for $4 \mathrm{~h}$. Almost $120 \mathrm{~g}$ of each oil was added to the first column slowly and gradually. A vacuum pump with high pressure was utilized to facilitate oil withdrawal from the chromatography column. The contents of the output from the first column was transferred to the second column and this operation was repeated once more. The purified samples were maintained at $-18{ }^{\circ} \mathrm{C}$ (for a maximum period of two weeks) and the headspace was filled with 
nitrogen. According to previous research, this method can remove or significantly diminish tocopherols, phenolic compounds and metal elements. ${ }^{21}$

\section{CMC of LEC in sunflower oil}

129

130

Tetracyanoquinodimethane (TCNQ) was used as a reagent to measure the CMC of LEC. For this purpose, a blend of TCNQ and purified sunflower oil (with the ratio of 1:1, v/w) was made to contain $0.015-0.2 \%$ LEC. This blend was vortexed for $5 \mathrm{~h}$ by a magnetic stirrer at ambient temperature. To remove the TCNQ excess, the blend was centrifuged at $2000 \times \mathrm{g}$ for $15 \mathrm{~min}$. The supernatant was carefully collected and the absorbance was measured at $480 \mathrm{~nm}$ by a spectrophotometer. The standard curve was plotted using the LEC concentration vs. TCNQ absorption, and the tangent method was employed to calculate coordinates that demonstrated the CMC of LEC. ${ }^{22}$

\section{Preparation of inhibited peroxidation}

The peroxidation process of sunflower oil involved using a dry oven at $60{ }^{\circ} \mathrm{C}$. Briefly, $6 \mathrm{~g}$ of purified oil was added to a Petri dish $(6 \mathrm{~cm}$ diameter $)$ to provide a thin layer of oil. This condition causes the peroxidation rate not to be affected by the oxygen concentration. To prepare inhibited peroxidation, $0.33 \mathrm{mM}$ of each antioxidant was dissolved in $1 \mathrm{~mL}$ of acetone, and was added to the lipid substrate. The added solvent was eventually eliminated by nitrogen gas. To provide samples containing LEC, $6.60 \mathrm{mM}$ of LEC (molar weight: $758.1 \mathrm{~g} \mathrm{M}^{-1}$ ) (ratio $1: 10 \mathrm{w} / \mathrm{v}$ ) was dissolved in ethyl acetate for $1 \mathrm{~h}$ at $40{ }^{\circ} \mathrm{C}$ by a magnetic thermo-stirrer. Then, the purified oil was slowly added to the cooled solution and the stirring process remained at ambient temperature for $10 \mathrm{~min}$. In the next step, the added solvent was removed by a rotary evaporator. Finally, the peroxidation process (as mentioned above) was also repeated for samples containing LEC. $^{23}$

\section{$\log \mathbf{P}$}


The partition coefficients of the antioxidants under study, including their solubility ratio in a nonpolar to polar environment as $\log P$, were computed using ChemDraw software (version 16 Professional; PerkinElmer, Waltham, MA, USA).

\section{Monitoring the accumulation of ROOHs}

This process was carried out by sampling treatments under the peroxidation at certain time intervals. Then, the peroxide value was measured by a spectrophotometer according to Shanta and Decker (1994). For this purpose, regarding the peroxidation progress between 0.001-0.3 $\mathrm{g}$ of oil sample, the oil was weighed in $15 \mathrm{~mL}$ test tubes. Then, $9.8 \mathrm{~mL}$ chloroform-methanol $(7: 3, v / v)$ was added to the oil samples. Fifty $\mu \mathrm{L}$ of ammonium thiocyanate aqueous solution $(30 \%, w / v)$ was added to the oil sample and shaked for 5 seconds. The next stage involved mixing $50 \mu \mathrm{L}$ Iron (II) chloride solution $\left(\left[0.25 \mathrm{~g} \mathrm{FeSO}_{4} .7 \mathrm{H}_{2} \mathrm{O}\right.\right.$ dissolved in $\left.25 \mathrm{~mL} \mathrm{H}_{2} \mathrm{O}\right]+[0.2$ g barium chloride dehydrate dissolved in $\left.25 \mathrm{~mL} \mathrm{H}_{2} \mathrm{O}\right]+1 \mathrm{~mL} \mathrm{HCl} 10 \mathrm{~N}$, and then the resultant solution was filtered to remove barium sulphate deposits). After 5 minutes, the sample absorption was determined at $500 \mathrm{~nm} .{ }^{24}$ Eventually, the results were reported based on milliequivalent of oxygen per $\mathrm{kg}$ of oil $\left(\mathrm{meq} \mathrm{kg}{ }^{-1}\right)$ or molarity $\left(1 \mathrm{meq} \mathrm{kg}{ }^{-1}=0.504 \mathrm{mM}\right){ }^{25}$

\section{Kinetic parameters}

As shown in Figure 1, various kinetic parameters were obtained by plotting changes in ROOHs vs. time. Several equations were used in calculating these parameters, according to the following: 2,6

The oxidation reaction rate in the initiation phase can be expressed by Eq. (1):

$$
\frac{\mathrm{d}[\mathrm{ROOH}]}{\mathrm{dt}}=k_{1}
$$

where $k_{1}$ is the rate constant of the initiation phase. Eq. (2) is obtained by integrating Eq. (1) vs. the limited time from zero time to IP point, and a concentration range from $[\mathrm{ROOH}]_{0}$ (ROOHs amount at $\mathrm{t}=0)$ to $[\mathrm{ROOH}]_{\mathrm{IP}}$ : 


$$
[\mathrm{ROOH}]_{\mathrm{IP}}=[\mathrm{ROOH}]_{0}+k_{1} \mathrm{IP}
$$

173 As mentioned earlier, to evaluate the behavior of vegetable oils against oxidation and, 174 particularly, in the presence of antioxidants, a combination model was employed according to 175 Eq. (3). Based on the pseudo-first-order reaction, the formation rate of ROOHs in the 176 propagation phase $\left(k_{\mathrm{f}}\right)$ were expressed by Eq. (3):

$$
\frac{\mathrm{d}[\mathrm{ROOH}]}{\mathrm{dt}}=k_{\mathrm{f}}[\mathrm{ROOH}]
$$

177 after integration:

$$
[\mathrm{ROOH}]=\exp \left(k_{\mathrm{f}} \mathrm{t}-\mathrm{a}_{\mathrm{f}}\right)
$$

178 where af is the integration constant. Also, Eq. (5) finds rate constant of the ROOHs 179 decomposition $\left(k_{\mathrm{d}}\right)$ in the pseudo-second-order reaction:

$$
-\left(\frac{\mathrm{d}[\mathrm{ROOH}]}{\mathrm{dt}}\right)=k_{\mathrm{d}}[\mathrm{ROOH}]^{2}
$$

180 after integration:

$$
[\mathrm{ROOH}]=\frac{1}{k_{\mathrm{d}} \mathrm{t}-\mathrm{a}_{\mathrm{d}}}
$$

181 where $a_{d}$ is integration constant. By merging Eq. (3) and Eq. (5), we have:

$$
\frac{\mathrm{d}[\mathrm{ROOH}]}{d t}=k_{\mathrm{f}}[\mathrm{ROOH}]-k_{\mathrm{d}}[\mathrm{ROOH}]^{2}
$$

182 the integration of Eq. (7) gives:

$$
[\mathrm{ROOH}]=\frac{\mathrm{k}_{\mathrm{f}}}{\exp \left[\mathrm{k}_{\mathrm{f}}(\mathrm{a}-\mathrm{t})\right]+\mathrm{k}_{\mathrm{d}}}
$$

where a is an overall integration constant. Also, $[\mathrm{ROOH}]_{\max }$ is calculable by the following 184 equation:

$$
[\mathrm{ROOH}]_{\max }=\lim _{\mathrm{t} \rightarrow \infty}\left(\frac{\mathrm{k}_{\mathrm{f}}}{\exp \left[\mathrm{k}_{\mathrm{f}}(\mathrm{a}-\mathrm{t})\right]+\mathrm{k}_{\mathrm{d}}}\right)
$$


185 Eq. (8) is an empirical sigmoidal model that can be used for predicting the general trend of 186 oxidation reaction by having a turning point in the middle of the propagation phase. The 187 maximum achievable rate as $\mathrm{M}_{\mathrm{R}}$ in this point can be obtained by Eq. (10):

$$
\mathrm{M}_{\mathrm{R}}=\left(\frac{\mathrm{d}[\mathrm{ROOH}]}{\mathrm{dt}}\right)_{\max }=\frac{k_{\mathrm{f}}{ }^{2}}{4 \mathrm{k}_{\mathrm{d}}}
$$

188 The normalized form $\left(\mathrm{N}_{\mathrm{M}_{\mathrm{R}}}\right)$ of the $\mathrm{M}_{\mathrm{R}}$ can be obtained by Eq. (11):

$$
\mathrm{N}_{\mathrm{M}_{\mathrm{R}}}=\frac{\mathrm{M}_{\mathrm{R}}}{[\mathrm{ROOH}]_{\max }}
$$

189 The coordinates of the turning point are calculated by the following equations:

$$
\begin{aligned}
& \mathrm{t}_{\mathrm{M}_{\mathrm{R}}}=\frac{k_{\mathrm{f}} \mathrm{a}-\ln k_{\mathrm{d}}}{k_{\mathrm{f}}} \\
& {[\mathrm{ROOH}]_{\mathrm{M}_{\mathrm{R}}}=\frac{k_{\mathrm{f}}}{2 k_{\mathrm{d}}}}
\end{aligned}
$$

190 The x-coordinate of the IP point in the combination model can be obtained by integrating Eq.

191 (2) with Eq. (8) by the following equation:

$$
\mathrm{IP}=\frac{k_{\mathrm{f}}\left(2-k_{\mathrm{f}} \mathrm{a}+\ln k_{\mathrm{d}}\right)-4[\mathrm{ROOH}]_{0} k_{\mathrm{d}}}{4 k_{1} k_{\mathrm{d}}-k_{\mathrm{f}}^{2}}
$$

192 The end time of the propagation phase (EtPP) and propagation period (PP) are calculated by the 193 following equations:

$$
\begin{aligned}
& \mathrm{Et}_{\mathrm{PP}}=\frac{4 k_{\mathrm{d}} \mathrm{M}_{\mathrm{R}}-k_{\mathrm{f}} \mathrm{N}_{\mathrm{M}_{\mathrm{R}}}\left(2-k_{\mathrm{f}} \mathrm{a}+\ln k_{\mathrm{d}}\right)}{4 k_{\mathrm{d}} \mathrm{M}_{\mathrm{R}} \mathrm{N}_{\mathrm{M}_{\mathrm{R}}}} \\
& \mathrm{PP}=\mathrm{Et}_{\mathrm{PP}}-\mathrm{IP}
\end{aligned}
$$

$$
E=\frac{\mathrm{IP}_{A}}{\mathrm{IP}_{\mathrm{C}}}
$$


where $\mathrm{IP}_{\mathrm{C}}$ and $\mathrm{IP}_{\mathrm{A}}$ are the IPs in the absence and the presence of the antioxidant, respectively.

The ratio of the oxidation rate $\left(R_{\mathrm{or}}\right)$ is obtained as a measure of antioxidant strength $\left(1 / R_{\mathrm{or}}\right)$,

$$
R_{\text {or }}=\frac{k_{1_{\mathrm{A}}}}{k_{1_{\mathrm{C}}}}
$$

198

The oxidation resistance in the initiation phase $\left(O_{R}\right)$ and synergistic effect (SE) of LEC was 202

$$
\begin{aligned}
& O_{R}=\frac{I P}{k_{1}} \\
& S E(\%)=\left(1-\frac{O_{R_{A}}+O_{R_{L}}-2 O_{R_{C}}}{2\left(O_{R_{A}+L}-O_{R_{C}}\right)}\right) \times 100
\end{aligned}
$$

where $O_{R_{A}}, O_{R_{L}}, O_{R_{C}}$, and $O_{R_{A+L}}$ are oxidation sensitivity parameters of the antioxidant per se, LEC per se, control, and antioxidant + LEC, respectively.

\section{Water content}

The amounts of water being produced during the peroxidation process were measured by a Karl Fischer titrator device (KF Titrando, Metrohm, Herisau, Switzerland) in accordance with the manufacturer's guidelines.

\section{Particle size}

210 The changes in size and distribution of particles with peroxidation progress were analysed by dynamic light scattering (DLS) (SZ-100 nanopartica series, Horiba Ltd., Kyoto, Japan) at a scattering angle of $173^{\circ}$ and $25^{\circ} \mathrm{C}$. 


\section{Statistical analysis}

214 All tests were performed in three independent experiments and the results entered the analysis of variance. Statistical and regression analyses were performed using SPSS, CurveExpert, and Microsoft Office Excel software. Significant differences among the mean values were determined by Duncan's multiple range test $(P<0.05)$.

\section{Results and discussion}

\section{Evaluating primary kinetic parameters}

The predicted sigmoidal model fitted well on the curve of ROOHs production and distinguished the different phases of the oxidation process $\left(\mathrm{R}^{2} \geq 0.98\right)$. As shown in Figure $2 \mathrm{a}$, the longest duration of the initiation phase was recorded in samples containing LEC. Among them, the highest level was found in the FRA, followed by EFR and GOR. The exact values of IPs were listed (Table 1). Remarkable differences were observed in the performance of the antioxidants under study, although their phenolic rings were similar while being directly involved in displaying the antioxidant activity. Evaluating the $E$ parameter, as a symbol to introduce the hydrogen donating mechanism, ${ }^{26,27}$ revealed a significant increase in this factor. These results indicate the participation of antioxidant molecules in chain termination reaction as shown in the following equation: ${ }^{2}$

$$
\mathrm{AH}+\mathrm{ROO}^{\circ} \rightarrow \mathrm{ROOH}+\mathrm{A}^{*}
$$

During the oxidation process, lipid systems can produce a variety of free radicals with different redox potentials $\left(\mathrm{E}_{\mathrm{h}}\right)$ such as alkyl $\left(\mathrm{R}^{\circ}: 600 \mathrm{mV}\right)$, alkoxyl $\left(\mathrm{RO}^{\circ}: 1600 \mathrm{mV}\right)$, peroxyl (ROO`: $1000 \mathrm{mV}$ ), and hydroxyl ('OH: $2320 \mathrm{mV}) \cdot{ }^{28,}{ }^{29}$ In the beginning of the oxidation process, the only pathway of ROOHs production is the conversion of $\mathrm{R}^{\circ}$ to $\mathrm{ROO}^{\circ}$ (due to its low $\mathrm{E}_{\mathrm{h}}$ ) and its attack on the hydrogen attached to allylic or bis-allylic carbon. ${ }^{9}$ Thus, Eq. (22) is the first and 
the most important defense barrier generated by antioxidant molecules. The $E$ parameter led to results that indicated a higher efficiency of FRA in the hydrogen donating mechanism, compared to that in EFR and GOR (Table 1). However, as oxidation progressed, the pathway of reactions changed due to increasing $\mathrm{ROOH}$ molecules. These molecules attack the lipid substrate and contribute to the production of water, as evidenced by Eq. $(23),{ }^{20}$ thereby playing a key role in the oxidation process and in the performance of antioxidants:

$$
\mathrm{ROOH}+\mathrm{RH} \rightarrow \mathrm{RO}^{\bullet}+\mathrm{H}_{2} \mathrm{O}+\mathrm{R}^{\bullet}
$$

As shown in Table 2, in the control sample, the amount of water increased in production during the peroxidation process, as attributed to Eq. (23). However, in the presence of antioxidants, the amount of water production increased dramatically, compared to the control sample. This can be ascribed to the consumption of a part of antioxidant molecule in side reactions of the initiation chain, which produces water as shown in Eq. (24):

$$
\mathrm{AH}+\mathrm{ROOH} \rightarrow \mathrm{A}^{\bullet}+\mathrm{H}_{2} \mathrm{O}+\mathrm{RO}^{\bullet}
$$

A previous study indicated that GOR participates in this reaction. ${ }^{30}$ Accordingly, it is a logical assumption that other antioxidants i.e. FRA and EFR can also take part in the Eq. (24) due to identical phenolic rings.

As shown in Table 1, the antioxidants were able to reduce the $k_{1}$ and its derived index (i.e. $R_{\mathrm{or}}$ ), compared to non-inhibited peroxidation. The $R_{\text {or }}$ is a symbol of electron transfer mechanism and it reflects variations in antioxidant radical $\left(\mathrm{A}^{\circ}\right)$ performance. ${ }^{26,27}$ Thus, the FRA with the lowest value of $R_{\text {or }}$ showed the highest participation in quenching ROO` by producing A-OOR. Generally, by integrating the results that pertained to the two mentioned mechanisms, the best antioxidant activity was observed in FRA, followed by EFR, and GOR (Table 1, $A$ parameter). It is best to present a brief description of the achievements of LEC presence prior to discussing the behavior of antioxidants. 
The CMC of LEC is a criterion for introducing the maximum usable concentration beyond which LEC begins to self-aggregate and lose its effectiveness. ${ }^{22}$ By the TCNQ method, the CMC of LEC was calculated as $12.71 \mathrm{mM}$ and approximately a half of this amount was added to the bulk oil (to produce a heterogeneous bulk oil) so as to demonstrate its physicochemical properties. The addition of LEC to the functional environment of the antioxidants caused remarkable changes in the antioxidant performance (Table 1). As expected, LEC showed a limited antioxidant activity, but its synergistic effects were much more prominent $(\sim 65 \%)$. In the presence of LEC, a considerable change occurred in the mechanism of hydrogen donating of the antioxidants, which ultimately prolonged the IP. In an apparent contradiction, however, the presence of LEC caused a slight increase in the $k_{\mathrm{i}}$ compared to the absence of LEC (basic state). This indicates that the efficiency of the electron transfer mechanism decreased slightly and that the $\mathrm{A}^{\cdot}$ participated in one or more of the propagation chain reactions, as shown in the following equations: ${ }^{27}$

$$
\begin{aligned}
& \mathrm{A}^{\bullet}+\mathrm{ROOH} \rightarrow \mathrm{AH}+\mathrm{ROO}^{\bullet} \\
& \mathrm{A}^{\bullet}+\mathrm{RH} \rightarrow \mathrm{AH}+\mathrm{R}^{\bullet} \\
& \mathrm{A}-\mathrm{OOR} \rightarrow \mathrm{AO}^{\bullet}+\mathrm{RO}^{\bullet} \\
& \mathrm{A}^{\bullet}+\mathrm{O}_{2} \rightarrow \mathrm{AOO}^{\bullet}
\end{aligned}
$$

The difference in the performance of antioxidants is likely due to the appropriate organization of the oxidative microreactors and the interaction of antioxidants with these structures, which is discussed in the following section.

\section{Interoperations of occurred events during the initiation phase}

For all samples, as the water content increased, the size of the reverse micelles kept increasing up to a point where the initiation phase ended (Table 2). In the presence of LEC, the size of reverse micelles increased more because of a greater reduction in interfacial tension. The effect 
of this behavior change is well observable in enhancing the micelles size at IP point (Table 2). Generally, the addition of LEC increased all kinetic parameters as shown in Figure $2 \mathrm{~b}$. Such behaviors probably arose from an increase in the number of oxidation microreactors, as understood from Span changes (Table 2). Considering the migration of oxidation products to these structures, the accessibility of all oxidation active components can increase to each other. However, the main factor for the movement of antioxidants toward these structures is the hydrophilic-lipophilic balance, which is evaluated by $\log \mathrm{p}$ (as a criterion of polarity). ${ }^{9,12,16}$ The values of $\log \mathrm{p}$ were computed in the case of FRA (1.42), EFR (2.02) and GOR (10.12). Significant differences between these values can cause a difference in the interfacial performance of these compounds. The simultaneous presence of carboxyl and hydroxyl groups in the chemical structure of FRA can act as a potent driving force to move FRA toward the interface. However, the attachment of hydrophobic compounds to the carboxylic group (i.e. ethyl or phytosteryl) can increase the solubility of these compounds in the bulk oil. Therefore, there would be a decrease in the tendency of these compounds to migrate toward the interface, along with a decrease in their interfacial performance (Figure $3 \mathrm{a}$ and $3 \mathrm{~b}$ ). The outcome of these events is a decrease in antioxidant activity. Obviously, the size of the attached hydrophobic group can play an important role in actualizing antioxidant activity. ${ }^{13}$ However, our results showed that this decrease in efficiency is not uniform when there is an increase in the alkyl chain of the antioxidants.

As mentioned earlier, the presence of LEC causes a remarkable increase in the $E$ factor of antioxidants $(\sim 55 \%)$. Also, the $E$ factor can enhance by increasing the antioxidant polarity. As shown in Figure 2c, the growth coefficient in the presence of LEC was higher than that of the basic state (1.143 vs. 1.131). Thus, the synergy of these two factors (i.e. antioxidant polarity and presence of LEC) can considerably excite the participation of antioxidants in the mechanism of hydrogen donating. On the other hand, reducing the polarity of antioxidants 
increased their $R_{\text {or }}$ factor, the growth coefficient of which was higher in the presence of LEC than its absence (0.881 vs. 0.868$)$. Furthermore, the addition of LEC increased the $R_{\text {or }}$ factor of antioxidants by $\sim 4 \%$, compared to the basic state. Therefore, antioxidants with lower polarity showed a higher degree of participation in equations (25-28), while the participation was generally more severe in the presence of LEC. Such behavior likely originated from the partitioning of antioxidant molecules or of their radicals between bulk oil and microreactors of oxidation. The behavior is probably a manifestation of their polarity. The ROOHs of sunflower oil are likely to move to the interface because of their higher polarity and a greater driving force which, in turn, is caused by the presence of at least two oxygen molecules on their allylic and/or bi-allylic carbon. ${ }^{31,} 32$ Therefore, the effective collisions decrease between less polar antioxidants and intermediate components of oxidation. As shown in Scheme 1, the inhibitory pathways of the antioxidants under study can be different. In fact, the relatively high energy of bound dissociation $(-\mathrm{OH})^{11}$ can make FRA and its derivatives unable to quench the $\mathrm{R}^{\circ}$, so that their only pathway in demonstrating antioxidant activity is the reaction with ROO'. Considering that the $\mathrm{ROO}^{\circ}$ is necessarily located in the interface, a lower level of access to these radicals ensues among antioxidants which lower polarity. Accordingly, the access of $\mathrm{A}^{*}$ to target free radicals is reduced as well. Thus, $\mathrm{A}^{\bullet}$ must either participate in the side reactions of the propagation chain or in the neutral reaction of the termination chain, i.e. the collision between the two radicals $\left(\mathrm{A}^{\bullet}+\mathrm{A}^{\bullet} \rightarrow\right.$ product $){ }^{33}$

\section{Key role of water in the oxidation process}

324 As can be deduced from Table 2, the increase in the size and number of reverse micelles is in parallel with the amount of water produced in the system. These water molecules have a high tendency to attach to the hydrophilic head of LEC for a decrease in interfacial tension. ${ }^{34}$ Therefore, the formation of reverse micelles likely accelerates by creating preliminary cores arising from water production. Obviously, the existing antioxidants in the environment will 
have a better chance to be deployed in the interface (where free radicals are located) by increasing the number of microreactors. ${ }^{20,32}$ As shown in Figure $2 \mathrm{~d}$, a desirable correlation was found between the amount of water being produced in the initiation phase of oxidation and the $E$ factor. This can prove that the hydrogen donating mechanism of antioxidants becomes more active by increasing the water content.

As oxidation progressed, the production of water and ROOHs increased uniformly, but the migration rate of water molecules into the core of the micelle probably occurred faster than the ROOHs to the interface. This difference in rate can be explained by the small size of water molecules and the high driving force. These events probably caused the core of the micelle to grow faster, so that existing surfactants and the resultant ROOHs became insufficient to cover this increase in volume. Thus, the reverse micelles disintegrated. The result of these events can be the transition the initiation phase to the propagation.

\section{Kinetic parameters of propagation and termination phases}

As listed in Table 2, the size of the reverse micelles significantly reduced after the IP point (AIP), and a relative physical stability was probably established in the system. However, the chemical reactions that happened thereafter were assumed to be completely different. Hemolytic decomposition (Eq. (29)) is one of the most important reactions that occurs during lipid peroxidation: ${ }^{1,6}$

$$
\mathrm{ROOH} \rightarrow{ }^{\bullet} \mathrm{OH}+\mathrm{RO}^{\bullet}
$$

The products of this reaction are very important. Given that the oxygen in the $\mathrm{RO}^{\bullet}$ usually mounts onto the bis-allylic carbon, the $\beta$-cleavage reaction occurs definitely because this type of radical is instable. It causes the surface-active compounds to be produced at a higher level than in the initiation phase. ${ }^{1}$ On the other hand, the hydroxyl radical that is produced according to Eq. (29) can attack each compound due to its high $\mathrm{E}_{\mathrm{h}}$ and, thus, can separate its $\mathrm{H}^{28,29}$ This 

to increase the micelles size.

$$
\mathrm{HO}^{\bullet}+\mathrm{RH} \text { (or any other similar compounds) } \rightarrow \mathrm{H}_{2} \mathrm{O}+\mathrm{R}^{\bullet}
$$

354 The collision of accumulated molecules of $\mathrm{ROOH}$ with each other is another reaction that occurs during the propagation phase. The product of this reaction, known as the bimolecular reaction, is water as shown in the following equation: ${ }^{20}$

$$
2 \mathrm{ROOH} \rightarrow \mathrm{ROO}^{\bullet}+\mathrm{H}_{2} \mathrm{O}+\mathrm{RO}^{\bullet}
$$

The water molecules produced by Eq. (30) and Eq. (31) usually play a very important role in the events that occur during the propagation phase.

A considerable difference was observed between the duration of PP in the inhibited peroxidation, compared to the non-inhibited condition (Table 1). Considering that the lipid substrate is composed of only pure triacylglycerols of sunflower oil, each event is a direct result of the antioxidant performance which is added to the system. Therefore, it is concluded that antioxidant molecules are not entirely consumed during the initiation phase of oxidation and their remnants indicate some antioxidant activity during the propagation phase. Moreover, the addition of LEC caused a tangible increase in the duration of PP, which is likely due to the physical role of this compound in inhibiting oxidation reactions. The onset of the propagation phase is associated with the regeneration of the reverse micelles and with an increase in their size through time (Table 2), meaning that the physical events in the initiation phase of oxidation are likely to reoccur in the propagation phase (Figure 3c). However, at this stage, the size of reverse micelles increased considerably due to the addition of a large volume of surface-active agents produced by the oxidation process. Obviously, all radicals have a strong tendency to migrate toward the reverse micelles, ${ }^{32}$ and the formation of reverse micelles is supported by the presence of LEC. Thus, the reverse micelles tend to multiply in number and enlarge, compared to the basic state (Table 2). As a result, the LEC causes a delay in the secondary 
breaking point of the reverse micelles, i.e. $\mathrm{ROOH}_{\max }$ (Table 1 and 2). In fact, the $\mathrm{ROOH}_{\max }$ is a secondary $\mathrm{CMC}$ in the peroxidation of oils, as it occurs precisely at the end of the propagation phase (Table 2). Through these events, the PP duration is prolonged. The results indicated that adding LEC reduced the amounts of $k_{\mathrm{f}}$ and $k_{\mathrm{d}}$ remarkably, compared to the control sample (Table 1) ( $k_{\mathrm{c}}: 2.87$ vs. $1.69 ; k_{\mathrm{d}}: 1.35$ vs. 0.64$)$. These results confirm the physical role of LEC in the inhibition of oxidation reactions in the propagation phase.

The results of the maximum rate of ROOHs formation $\left(\mathrm{M}_{\mathrm{R}}\right)$ showed that samples which had been treated with the antioxidant, compared to untreated samples, reached a lower rate at the turning point (Table 1). This can be attributed to the remaining molecules of the antioxidant in the propagation phase that may act as a barrier to the actualization of a maximum rate. Meanwhile, it should be considered that the maximum rate occurs within a specified concentration range of $\mathrm{ROOH}$, i.e. $[\mathrm{ROOH}]_{\mathrm{M}_{\mathrm{R}}}$ (Table 1). In the presence and absence of LEC, the average of these concentrations are equal to $127.51 \pm 4.94 \mathrm{meq} \mathrm{kg}^{-1}(\mathrm{LEC}+(\mathrm{FRA}+\mathrm{LEC})$ $+(\mathrm{EFR}+\mathrm{LEC})+(\mathrm{GOR}+\mathrm{LEC}))$ and $101.98 \pm 3.90 \mathrm{meq} \mathrm{kg}^{-1}(\mathrm{Control}+\mathrm{FRA}+\mathrm{EFR}+\mathrm{GOR})$ respectively.

$\mathrm{N}_{\mathrm{M}_{\mathrm{R}}}$ is a symbol of lipid resistance against propagation chain reactions, in which the lower values of this criterion indicate a higher resistance of the system. ${ }^{5}$ The results showed that adding LEC significantly reduced this parameter (Table 1). The end time of the propagation phase $\left(\mathrm{Et}_{\mathrm{PP}}\right)$ indicated that the highest and the lowest time in the inhibited peroxidation pertained to FRA + LEC and GOR, respectively (1721 vs. $897 \mathrm{~min}$ ).

Most oxidation products that are produced over time convert to other products due to their high reactivity and, thus, the trends of their production can fluctuate frequently. The water content is one of the most stable oxidation indices that can be produced during the oxidation process. ${ }^{20}$ The results showed that this parameter has various linear relationships with some oxidation parameters of the initiation phase or of the propagation phase. For example, Figure 
4a shows the relationship between one of the key parameters, namely the ratio of the maximum achievable concentration of ROOHs in the initiation phase to its rate constant $\left(\mathrm{ROOH}_{\mathrm{IP}} / k_{l}\right)$ and the water content at the IP point. This relationship shows that increasing the water content can significantly reduce the oil peroxidation rate at the initiation phase. Interestingly, the amount of water at the IP point can even change the overall trend of the oxidation process at the propagation phase. For example, Figure $4 \mathrm{~b}$ shows that multiplying the $k_{\mathrm{f}}$ with the ratio of the coordinates at the turning point $\left([\mathrm{ROOH}]_{\mathrm{M}_{\mathrm{R}}} / \mathrm{t}_{\mathrm{M}_{\mathrm{R}}}\right)$ correlates with the water content at the IP point. In addition, the water content being produced during the propagation phase effectively enhances the ratio of the maximum rate of $\mathrm{ROOHs}$ formation to the rate of their decomposition $\left(\mathrm{M}_{\mathrm{R}} / \mathrm{k}_{\mathrm{d}}\right)$ (Figure $\left.4 \mathrm{c}\right)$. This effect can be attributed to the overall reduction of the rate constant of ROOHs decomposition by increasing water content. Considering that the number of oxidation microreactors increases by increasing the water content, many sites would exist to enhance the overall capacity of receiving ROOHs. Thus, the probability of effective collisions between ROOHs decreases and, accordingly, their decomposition occurs more slowly. Generally, the results showed that the said events have several effects during the initiation phase of the oxidation. They could considerably affect the parameters in relation to the turning point. On the other hand, changing the position of time or concentration of the turning point can also affect the end time of the propagation phase. For instance, Figure $4 \mathrm{~d}$ shows a linear relationship between the antioxidant activity and the result of multiplying the maximum rate of ROOHs production with the occurrence time of the turning point $\left(\mathrm{M}_{R} \times t_{M_{R}}\right)$. Given that the $\mathrm{M}_{\mathrm{R}}$ of the antioxidants did not change much (Table 1), it can be concluded that improving the efficiency of antioxidants remarkably increases the occurrence time of the turning point. Figure $4 \mathrm{e}$ shows a linear relationship between the time/concentration coordinates of the turning point and the end time of the propagation phase. This result suggests that the delay in achieving the turning point can considerably increase the propagation period. The 
turning point in the middle of the propagation phase appears to be the point where the antioxidant activity becomes zero. In general, the set of Figure 4 proves that all of the events that occurred in the lipid oxidation process were interconnected like an intertwined chain.

\section{Conclusion}

The present research can considerably change the prospects for practical applications of relatively polar antioxidants. The paradigm selected in this study is a feedback about the use of antioxidants that are likely to migrate to the water-oil interface, despite having sufficient solubility in oil environments. Thus, a fundamental change can probably take place in using these antioxidants, so that adding specialized surfactants to oil environments in the presence of these antioxidants would remarkably increase their efficiency. Irrespective of the macro objectives of this project, our results revealed facts that had previously been less sought for in research. One of the most important achievements of this study was the identification of key roles of water production during the process of lipid oxidation, as a major, basic element in directing this process. Another achievement of this research explained in detail how physicochemical events occur during oil oxidation and how their role can assist in the evolution of this process. Our understanding of fundamental facts in relation to the oxidation process is still insignificant, although the present study can be an inspiring step forward.

\section{References}

1. Shahidi, F. Bailey's Industrial Oil and Fat Products. (6th ed.), Volume 1, Edible Oil and Fat Products: Chemistry, Properties, and Health Effects, John Wiley and Sons, Hoboken, USA. (2005). 
2. Kamal-Eldin, A. \& Yanishlieva, N. Kinetic analysis of lipid oxidation data. In A.

Kamal-Eldin, A.; Pokorny, J. (Eds.). Analysis of lipid oxidation. Champaign, Illinois: AOCS Press, pp. 234-263 (2005).

3. Shim, S. D. \& Lee, S. J. Shelf-life prediction of perilla oil by considering the induction period of lipid oxidation. Eur. J. Lipid Sci. Tech. 113, 904-909 (2011).

4. Farhoosh, R. Reliable determination of the induction period and critical reverse micelle concentration of lipid hydroperoxides exploiting a model composed of pseudo-first and -second order reaction kinetics. LWT - Food Sci. Tech. 98, 406-410 (2018).

5. Pinchuk, I. \& Lichtenberg, D. Analysis of the kinetics of lipid peroxidation in terms of characteristic time-points. Chem. Phys. Lipids. 178, 63-76 (2014).

6. Farhoosh, R. Critical kinetic parameters and rate constants representing lipid peroxidation as affected by temperature. Food Chem. 340, 128137 (2021).

7. Farhoosh, R., Johnny, S., Asnaashari, M., Molaahmadibahraseman, N. \& Sharif, A. Structure-antioxidant activity relationships of o-hydroxyl, o-methoxy, and alkyl ester derivatives of p-hydroxybenzoic acid. Food Chem. 194, 128-134 (2016).

8. Stewart, et al. Effects of intramolecular hydrogen bonding and sterically forced noncoplanarity on organic donor/acceptor two-photon-absorbing molecules. Phys. Chem. Chem. Phys. 20, 19398-19407 (2018).

9. Frankel, E.N. Antioxidants. Lipid Oxidation, 2nd Edition. Elsevier publications. pp. 99127 (2005).

10. Xu, Z. \& Godber, J. S. Purification and identification of components of $\gamma$-oryzanol in rice bran oil. J. Agric. Food Chem. 47, 2724-2728 (1999).

11. Nenadis, N. \& Zhang, H. Y. Tsimidou, M.Z. Structure-Antioxidant Activity J. Agric. Food Chem. 51, 1874-1879 (2003). 
12. Laguerre, M. et al. What makes good antioxidants in lipid-based systems? The next

474

475

476

477

478

479

480

481

482

483

484

485

486

487

488

489

490

491

492

493

494

495

496

497 theories beyond the polar paradox. Crit. Rev. Food Sci. Nutr. 55, 183-201 (2013).

13. Budilarto, E. S. \& Kamal-Eldin, A. The supramolecular chemistry of lipid oxidation and antioxidation in bulk oils. Eur. J. Lipid Sci. Technol. 117(8), 1095-1137 (2015).

14. Chen, B. C., Han, A., McClements, D. J. \& Decker, E. A. Physical structures in soybean oil and their impact on lipid oxidation. J. Agric. Food Chem. 58, 11993-11999 (2010).

15. Lehtinen, O. P. et al. Effect of temperature, water content and free fatty acid on reverse micelle formation of phospholipids (especially lecithin) in vegetable oil. Colloids Surf. B. 160, 355-363 (2017).

16. Laguerre, M. Bily, A., Roller, M. \& Birtic, S. Mass transport phenomena in lipid oxidation and antioxidation. Annu. Rev. Food Sci. Technol. 8, 391-411 (2017).

17. Cardenia, V., Waraho, T., Rodriguez-Estrada, M., McClements, J. D. \& Decker, E.A. Antioxidant and prooxidant activity behavior of phospholipids in stripped soybean oil-in-water emulsions. J. Am. Oil Chem.' Soc. 88, 1409-1416 (2011).

18. Kittipongpittaya, K., Panya, A., McClements, J. D. \& Decker, E. Impact of free fatty acids and phospholipids on reverse micelles formation and lipid oxidation in bulk oil. J. Am. Oil Chem.' Soc. 91, 453-462 (2014).

19. Oehlke, K., Heins, A., Stockmann, H. \& Schwarz, K. Impact of emulsifier microenvironments on acid-base equilibrium and activity of antioxidants. Food Chem. 118, 4855 (2010).

20. Budilarto, E. S. \& Kamal-Eldin, A. Water content and micelle size change during oxidation of sunflower and canola oils. Eur. J. Lipid Sci. Technol. 117(12), 1971-1977 (2015).

21. Toorani, M. R., Farhoosh, R., Golmakani, M. \& Sharif, A. Antioxidant activity and mechanism of action of sesamol in triacylglycerols and fatty acid methyl esters of sesame, olive, and canola oils. LWT - Food Sci. Tech. 103, 271-278 (2019). 

phosphoethanolamine reverse micelles on lipid oxidation in bulk oils. J. Am. Oil Chem.' Soc. 91, 1931-1937 (2014).

23. Ramadan, M. F. Quercetin increases antioxidant activity of soy lecithin in a triolein model system. LWT - Food Sci. Tech. 41, 581-587 (2008).

24. Shantha, N. C. \& Decker, E. A. Rapid, sensitive, iron-based spectrophotometric methods for determination of peroxide values of food lipids. J. AOAC Int. 77, 421-424 (1994). activity and mechanism of action of thymol and carvacrol in two lipid systems. Food Chem. 64, 59-66 (1999). radicals of inhibitors. Chem. Rev. 87(6), 1313-1357 (1987). in Lipid Oxidation: Lipid Oxidation Pathways, (Ed: A. Kamal-Eldin), AOCS Press, Champaign, pp. 85-110 (2003). (1988).

29. Koppenol, W. Oxyradical reactions: From bond-dissociation energies to reduction potentials. FEBS Lett. 264, 165-167 (1990).

30. Toorani, M. R., Golmakani, M. \& Hashemi-Gahruie, H. Antioxidant activity and inhibitory mechanism of $\gamma$-oryzanol as influenced by unsaturation degree of lipid systems. $L W T$ - Food Sci. Tech. 133, 109930 (2020). 
32. Ghnimi, S., Budilarto, E. \& Kamal-Eldin, A. The new paradigm for lipid oxidation

524 and insights to microencapsulation of omega-3 fatty acids. Compr. Rev. Food Sci. Food Saf. 16, 1206-1218 (2017).

33. Masuda, T., Yamada, K., Maekawa, T., Takeda, Y. \& Yamaguchi, H. Antioxidant Mechanism Studies on Ferulic Acid: Isolation and Structure Identification of the Main Antioxidation Product from Methyl Ferulate. Food Sci. Technol. Res. 12(3), 173-177 (2006). 34. Mansouri, H. Farhoosh, R. \& Rezaie, M. Interfacial performance of gallic acid and methyl gallate accompanied by lecithin in inhibiting bulk phase oil peroxidation. Food Chem. 328, 127128 (2020).

\section{Abbreviations}

$\boldsymbol{A}$ : antioxidant activity, a: integration constant of sigmoidal model, $\mathbf{A}^{\bullet}$ : antioxidant radical; AH: antioxidant molecule, AIP: after IP point, CMC: critical micelle concentration; $\boldsymbol{E}$ : effectiveness of antioxidant, EFR: ethyl ferulate, $\mathbf{E}_{\mathbf{h}}$ : redox potential, Etpp: end time of the termination phase, FRA: ferulic acid, GOR: $\gamma$-oryzanol, H: hydrogen, IP: induction period, $\boldsymbol{k}_{\mathbf{f}}$ : rate constant of hydroperoxides formation at the propagation phase, $\boldsymbol{k}_{\mathrm{d}}$ : rate constant of hydroperoxides decomposition at the propagation phase, $\boldsymbol{k}_{\mathbf{1}}$ : rate constant of the initiation phase, LEC: lecithin, Log P: partition coefficient, MR: maximum rate of hydroperoxides formation in the propagation phase, $\mathbf{N}_{\mathbf{M}_{\mathbf{R}}}$ : normalized form of maximum rate of hydroperoxides formation in the propagation phase, PP: propagation period, $\mathbf{O H}$ : hydroxyl group, ${ }^{\mathbf{O H}}$ hydroxyl radical, $\mathbf{R}^{\bullet}$ : alkyl radical, $\mathbf{R H}$ : lipid reactant, $\mathbf{R O}^{*}$ : alkoxyl radical, $\mathbf{R O O}^{\circ}$ : peroxyl radical, ROOHs: hydroperoxide(s), ROOHIP: the hydroperoxides concentration at IP point, $[\mathbf{R O O H}]_{\max }$ : maximum concentration of the produced hydroperoxides, $[\mathbf{R O O H}]_{\mathbf{M}_{\mathbf{R}}}$ : the hydroperoxides concentration in the point of maximum rate of hydroperoxides formation (or 
547 turning point), $\mathbf{R}_{\mathbf{o r}}$ : ratio of oxidation rate, TCNQ: Tetracyanoquinodimethane, $\mathbf{t}_{\mathbf{M}_{\mathbf{R}}}$ :

548 occurrence time of maximum rate of hydroperoxides formation.

\section{Acknowledgement}

550 This work was financially supported by Shiraz University.

551

552 Author contributions

553 M.R.T: Conceptualization, Data curation, Formal analysis, Investigation, Methodology,

554 Resources, Software, Validation, Visualization, Writing-original draft. M-T.G.: Funding

555 acquisition, Project administration, Supervision, Writing-review and editing.

556

557 Competing interests

558 The authors declare no conflict of interest.

559

560 Additional information

561 Correspondence and requests for materials should be addressed to R.F. 
Table 1. Kinetic parameters related to the initiation, propagation, and termination phases of the stripped sunflower oil peroxidation (Control) containing lecithin (LEC), ferulic acid (FRA), ethyl ferulate (EFR), $\gamma$-oryzanol (GOR), and their combinations at $60^{\circ} \mathrm{C}$.

\begin{tabular}{|c|c|c|c|c|c|c|c|c|c|}
\hline \multirow{2}{*}{ Sample } & \multicolumn{9}{|c|}{ Kinetic parameters related to initiation and propagation phases } \\
\hline & IP A & $E^{\text {в }}$ & $k_{1}\left(\times 10^{2}\right)^{\mathrm{c}}$ & $R_{\text {or }}{ }^{\mathrm{D}}$ & $A^{\mathrm{E}}$ & $\mathrm{ROOH}_{\mathrm{IP}}{ }^{\mathrm{F}}$ & PP G & $k_{f}\left(\times 10^{2}\right)^{\mathrm{H}}$ & $k_{d}\left(\times 10^{4}\right)^{\mathrm{I}}$ \\
\hline Control & $194 \pm 2^{\mathrm{h} *}$ & - & $9.00 \pm 0.05^{\mathrm{a}}$ & - & - & $18.2 \pm 0.3^{\mathrm{b}}$ & $127 \pm 5^{\mathrm{d}}$ & $2.87 \pm 0.01^{\mathrm{a}}$ & $1.35 \pm 0.01^{\mathrm{a}}$ \\
\hline LEC & $352 \pm 4^{\mathrm{g}}$ & $1.82 \pm 0.07^{\mathrm{g}}$ & $8.15 \pm 0.03^{b}$ & $0.91 \pm 0.00^{\mathrm{a}}$ & $2.0 \pm 0.2^{\mathrm{f}}$ & $29.4 \pm 0.3^{\mathrm{a}}$ & $210 \pm 9^{b}$ & $1.69 \pm 0.02^{\mathrm{d}}$ & $0.64 \pm 0.04^{\mathrm{d}}$ \\
\hline FRA & $934 \pm 9^{d}$ & $4.82 \pm 0.03^{\mathrm{d}}$ & $1.85 \pm 0.02^{\mathrm{h}}$ & $0.21 \pm 0.00^{\mathrm{g}}$ & $23.4 \pm 0.5^{\mathrm{b}}$ & $18.1 \pm 0.2^{\mathrm{b}}$ & $173 \pm 7^{\mathrm{c}}$ & $2.11 \pm 0.00^{\mathrm{c}}$ & $1.02 \pm 0.01^{\mathrm{c}}$ \\
\hline EFR & $765 \pm 7^{\mathrm{e}}$ & $3.95 \pm 0.03^{\mathrm{e}}$ & $2.29 \pm 0.01^{\mathrm{f}}$ & $0.25 \pm 0.00^{\mathrm{e}}$ & $15.5 \pm 0.2^{\mathrm{d}}$ & $18.3 \pm 0.5^{\mathrm{b}}$ & $168 \pm 10^{\mathrm{c}}$ & $2.16 \pm 0.01^{b}$ & $1.11 \pm 0.00^{\mathrm{b}}$ \\
\hline GOR & $730 \pm 5^{f}$ & $3.77 \pm 0.04^{\mathrm{f}}$ & $2.46 \pm 0.00^{\mathrm{d}}$ & $0.27 \pm 0.00^{c}$ & $13.7 \pm 0.2^{\mathrm{e}}$ & $18.7 \pm 0.3^{\mathrm{b}}$ & $167 \pm 4^{\mathrm{c}}$ & $2.16 \pm 0.00^{\mathrm{b}}$ & $1.08 \pm 0.01^{\mathrm{b}}$ \\
\hline $\mathrm{LEC}+\mathrm{FRA}$ & $1453 \pm 19^{a}$ & $7.50 \pm 0.07^{\mathrm{a}}$ & $1.96 \pm 0.01^{\mathrm{g}}$ & $0.22 \pm 0.00^{\mathrm{f}}$ & $34.4 \pm 0.6^{\mathrm{a}}$ & $29.2 \pm 0.2^{\mathrm{a}}$ & $269 \pm 12^{\mathrm{a}}$ & $1.32 \pm 0.01^{\mathrm{f}}$ & $0.50 \pm 0.00^{f}$ \\
\hline $\mathrm{LEC}+\mathrm{EFR}$ & $1202 \pm 10^{\mathrm{b}}$ & $6.20 \pm 0.04^{b}$ & $2.39 \pm 0.01^{\mathrm{e}}$ & $0.27 \pm 0.00^{\mathrm{d}}$ & $23.3 \pm 0.3^{b}$ & $29.5 \pm 0.5^{\mathrm{a}}$ & $251 \pm 5^{\mathrm{a}}$ & $1.40 \pm 0.00^{\mathrm{e}}$ & $0.56 \pm 0.01^{\mathrm{e}}$ \\
\hline $\mathrm{LEC}+\mathrm{GOR}$ & $1112 \pm 8^{c}$ & $5.74 \pm 0.03^{c}$ & $2.53 \pm 0.01^{\mathrm{c}}$ & $0.28 \pm 0.00^{\mathrm{b}}$ & $20.4 \pm 0.1^{\mathrm{c}}$ & $28.9 \pm 0.4^{\mathrm{a}}$ & $256 \pm 9^{a}$ & $1.38 \pm 0.00^{\mathrm{e}}$ & $0.56 \pm 0.00^{\mathrm{e}}$ \\
\hline
\end{tabular}

Continues kinetic parameters

\begin{tabular}{|c|c|c|c|c|c|c|c|c|}
\hline \multirow{2}{*}{ Sample } & \multicolumn{8}{|c|}{ Contınues kinetic parameters } \\
\hline & {$[\mathrm{ROOH}]_{\max }{ }^{\mathrm{J}}$} & $\mathrm{M}_{\mathrm{R}}{ }^{\mathrm{K}}$ & $\mathrm{N}_{\mathrm{M}_{\mathrm{R}}}\left(\times 10^{3}\right)^{\mathrm{L}}$ & {$[\mathrm{ROOH}]_{\mathrm{M}_{\mathrm{R}}}{ }^{\mathrm{M}}$} & $t_{M_{R}}{ }^{N}$ & $\mathrm{a}^{\mathrm{o}}$ & $\mathrm{Et}_{\mathrm{PP}}{ }^{\mathrm{P}}$ & $O_{R}\left(\times 10^{3}\right)^{\mathrm{Q}}$ \\
\hline Control & $214 \pm 8^{\mathrm{c} *}$ & $1.53 \pm 0.02^{\mathrm{a}}$ & $7.18 \pm 0.02^{\mathrm{a}}$ & $107 \pm 2^{b}$ & $251 \pm 5^{\mathrm{h}}$ & $-59 \pm 2^{e}$ & $321 \pm 7^{\mathrm{h}}$ & $2.15 \pm 0.2^{\mathrm{g}}$ \\
\hline FRA & $206 \pm 5^{\mathrm{c}}$ & $1.09 \pm 0.01^{\mathrm{b}}$ & $5.27 \pm 0.02^{\mathrm{c}}$ & $103 \pm 3^{b}$ & $1012 \pm 21^{\mathrm{d}}$ & $576 \pm 31^{\mathrm{bc}}$ & $1107 \pm 10^{\mathrm{d}}$ & $50.3 \pm 0.4^{\mathrm{b}}$ \\
\hline EFR & $195 \pm 4^{\mathrm{c}}$ & $1.06 \pm 0.02^{\mathrm{b}}$ & $5.40 \pm 0.03^{b}$ & $98 \pm 5^{b}$ & $840 \pm 13^{\mathrm{e}}$ & $419 \pm 24^{\mathrm{d}}$ & $933 \pm 16^{\mathrm{e}}$ & $33.4 \pm 0.5^{\mathrm{d}}$ \\
\hline GOR & $200 \pm 7^{c}$ & $1.08 \pm 0.00^{\mathrm{b}}$ & $5.41 \pm 0.01^{\mathrm{b}}$ & $100 \pm 6^{b}$ & $805 \pm 8^{\mathrm{f}}$ & $383 \pm 15^{\mathrm{d}}$ & $897 \pm 7^{\mathrm{f}}$ & $29.6 \pm 0.3^{\mathrm{e}}$ \\
\hline $\mathrm{LEC}+\mathrm{EFR}$ & $249 \pm 9^{a b}$ & $0.87 \pm 0.00^{\mathrm{c}}$ & $3.50 \pm 0.02^{\mathrm{e}}$ & $125 \pm 4^{\mathrm{a}}$ & $1311 \pm 10^{\mathrm{b}}$ & $613 \pm 22^{\mathrm{b}}$ & $1453 \pm 13^{b}$ & $50.2 \pm 0.7^{\mathrm{b}}$ \\
\hline $\mathrm{LEC}+\mathrm{GOR}$ & $244 \pm 5^{b}$ & $0.84 \pm 0.01^{\mathrm{d}}$ & $3.45 \pm 0.02^{\mathrm{e}}$ & $122 \pm 5^{\mathrm{a}}$ & $1222 \pm 17^{\mathrm{c}}$ & $513 \pm 26^{c}$ & $1367 \pm 17^{c}$ & $44.0 \pm 0.8^{c}$ \\
\hline
\end{tabular}

* In each column and in each section, means ( \pm standard deviation) with different lowercase letters are significantly different $(P<0.05) .{ }^{\mathrm{A}}$ Induction period (min), ${ }^{\mathrm{B}}$ effectiveness, ${ }^{\mathrm{C}}$ initiation rate constant $\left(\mathrm{meq} \mathrm{kg}{ }^{-1} \mathrm{~min}^{-1}\right),{ }^{\mathrm{D}}$ ratio of the oxidation rate, ${ }^{\mathrm{E}}$ antioxidant activity, ${ }_{\mathrm{F}}^{\mathrm{F}}$ the hydroperoxides concentration at the induction period $\left(\right.$ meq $\left.\mathrm{kg}^{-1}\right),{ }^{\mathrm{G}}$ propagation period (min), ${ }^{\mathrm{H}}$ rate constant of the hydroperoxide formation in the propagation phase $\left(\mathrm{min}^{-1}\right)$, ${ }^{\mathrm{I}}$ rate constant of the hydroperoxide decomposition in the propagation phase (meq $\left.\mathrm{kg}^{-1} \min ^{-1}\right),{ }^{\mathrm{J}}$ maximum of the hydroperoxides concentration (meq kg${ }^{-1}$ ), ${ }^{\mathrm{K}}$ maximum rate of the hydroperoxide formation (meq $\mathrm{kg}^{-1}$ min ${ }^{-1}$ ), ${ }^{\mathrm{L}}$ normalized form of the maximum rate $\left(\mathrm{min}^{-1}\right),{ }^{\mathrm{M}}$ the hydroperoxides concentration at the turning point $\left(\mathrm{meq} \mathrm{kg} \mathrm{k}^{-1}\right){ }^{\mathrm{N}}$ the occurrence time of the turning point (min), ${ }^{\mathrm{O}}$ overall integration constant $\left(\mathrm{kg}^{\mathrm{meq}} \mathrm{q}^{-1}\right),{ }^{\mathrm{P}}$ end time of the propagation phase $(\mathrm{min}),{ }^{\mathrm{Q}}$ the oxidation resistance $\left(\mathrm{min} / \mathrm{meq} \mathrm{kg}^{-1}\right)$. 
570 Table 2. Water content and reverse micelles size related to the stripped sunflower oil peroxidation (Control) containing lecithin (LEC), ferulic 571 acid (FRA), ethyl ferulate (EFR), $\gamma$-oryzanol (GOR), and their combinations at $60{ }^{\circ} \mathrm{C}$.

\begin{tabular}{|c|c|c|c|c|c|c|c|c|c|c|c|}
\hline \multirow[t]{3}{*}{ Sample } & \multirow{2}{*}{\multicolumn{5}{|c|}{ Water content $\left(\mu \mathrm{g} \mathrm{g}^{-1}\right)$}} & \multicolumn{6}{|c|}{ Reverse micelles } \\
\hline & & & & & & \multicolumn{5}{|c|}{ Particle size $\left(\times 10^{-2}\right)(\mathrm{nm})$} & \multirow[t]{2}{*}{ Span $\dagger$} \\
\hline & $\mathrm{BIO}$ & IP & AIP & PP & APP & $\mathrm{BIO}$ & IP & AIP & $\mathrm{pp}$ & APP & \\
\hline LEC & $199 \pm 25^{\mathrm{Ea}}$ & $245 \pm 35^{\text {Dg }}$ & $719 \pm 44^{\mathrm{Cc}}$ & $1425 \pm 49^{\mathrm{Be}}$ & $1601 \pm 41^{\mathrm{Ae}}$ & $1.94 \pm 0.10^{\mathrm{Ea}}$ & $18.38 \pm 1.04^{\mathrm{Cd}}$ & $5.11 \pm 0.33^{\mathrm{Dc}}$ & $49.38 \pm 0.60^{\mathrm{Ac}}$ & $25.38 \pm 1.27^{\mathrm{Bc}}$ & $1.57 \pm 0.12^{\mathrm{c}}$ \\
\hline FRA & $125 \pm 12^{\mathrm{Eb}}$ & $442 \pm 21^{\mathrm{Dd}}$ & $741 \pm 28^{\mathrm{Cc}}$ & $1898 \pm 70^{\mathrm{Bc}}$ & $2040 \pm 62^{\mathrm{Ac}}$ & $1.00 \pm 0.22^{\mathrm{Ec}}$ & $12.06 \pm 0.46^{\mathrm{Ce}}$ & $4.96 \pm 0.40^{\mathrm{Dc}}$ & $37.31 \pm 1.04^{\mathrm{Ad}}$ & $20.20 \pm 0.90^{\mathrm{Bd}}$ & $1.39 \pm 0.04^{\mathrm{c}}$ \\
\hline EFR & $133 \pm 18^{\mathrm{Eb}}$ & $322 \pm 4^{\mathrm{De}}$ & $697 \pm 19^{\mathrm{Cc}}$ & $1711 \pm 62^{\mathrm{Bd}}$ & $1825 \pm 37^{\mathrm{Ad}}$ & $1.25 \pm 0.18^{\mathrm{Ebc}}$ & $10.27 \pm 0.61^{\mathrm{Cf}}$ & $3.24 \pm 0.33^{\mathrm{Dd}}$ & $32.24 \pm 1.29^{\mathrm{Ae}}$ & $17.44 \pm 0.70^{\mathrm{Be}}$ & $1.33 \pm 0.04^{\mathrm{d}}$ \\
\hline GOR & $119 \pm 14^{\mathrm{Eb}}$ & $295 \pm 1^{\mathrm{Df}}$ & $715 \pm 24^{\mathrm{Cc}}$ & $1606 \pm 56^{\mathrm{Bd}}$ & $1752 \pm 44^{\mathrm{Ad}}$ & $1.51 \pm 0.21^{\mathrm{Eab}}$ & $9.62 \pm 0.67^{\mathrm{Cf}}$ & $2.15 \pm 0.41^{\mathrm{De}}$ & $29.88 \pm 0.77^{\mathrm{Ae}}$ & $16.21 \pm 1.24^{\mathrm{Be}}$ & $1.29 \pm 0.06^{\mathrm{d}}$ \\
\hline LEC + FRA & $174 \pm 27^{\mathrm{Ea}}$ & $817 \pm 24^{\mathrm{Da}}$ & $1512 \pm 25^{\mathrm{Ca}}$ & $2980 \pm 69^{\mathrm{Ba}}$ & $3246 \pm 95^{\text {Aa }}$ & $1.93 \pm 0.25^{\mathrm{Ea}}$ & $29.33 \pm 0.68^{\mathrm{Ca}}$ & $9.15 \pm 0.58^{\mathrm{Da}}$ & $58.12 \pm 0.95^{\mathrm{Aa}}$ & $32.75 \pm 036^{\mathrm{Ba}}$ & $1.84 \pm 0.05^{\mathrm{a}}$ \\
\hline $\mathrm{LEC}+\mathrm{EFR}$ & $186 \pm 11^{\mathrm{Ea}}$ & $709 \pm 10^{\mathrm{Db}}$ & $1405 \pm 33^{\mathrm{Cb}}$ & $2774 \pm 71^{\mathrm{Bb}}$ & $2955 \pm 39^{\mathrm{Ab}}$ & $1.77 \pm 0.21^{\mathrm{Ea}}$ & $25.91 \pm 0.41^{\mathrm{Cb}}$ & $7.03 \pm 0.63^{\mathrm{Db}}$ & $52.70 \pm 1.23^{\mathrm{Ab}}$ & $28.04 \pm 1.00^{\mathrm{Bb}}$ & $1.66 \pm 0.07^{\mathrm{ab}}$ \\
\hline $\mathrm{LEC}+\mathrm{GOR}$ & $161 \pm 26^{\mathrm{Ea}}$ & $631 \pm 26^{\mathrm{Dc}}$ & $1397 \pm 40^{\mathrm{Cb}}$ & $2635 \pm 64^{\mathrm{Bb}}$ & $2809 \pm 77^{\mathrm{Ab}}$ & $2.03 \pm 0.36^{\mathrm{Ea}}$ & $24.14 \pm 0.50^{\mathrm{Cc}}$ & $7.55 \pm 0.70^{\mathrm{Db}}$ & $49.17 \pm 0.92^{\mathrm{Ab}}$ & $26.12 \pm 1.14^{\mathrm{Bb}}$ & $1.60 \pm 0.04^{\mathrm{ab}}$ \\
\hline
\end{tabular}

* In each row and in each section, averages ( \pm standard deviation) with different uppercase letters are significantly different $(P<0.05)$. In each column,

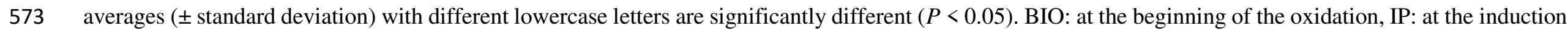

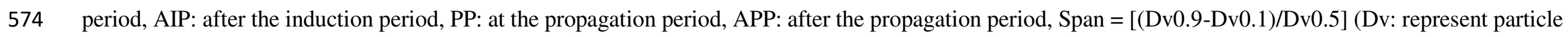

575 sizes larger than 10,50 , and $90 \%$ of the population), ${ }^{\dagger}$ the average spans of BIO, IP, AIP, PP, and APP. 
577 Figure 1. Schematic curve of hydroperoxides (ROOHs) production and a guide of calculated 578 kinetic points. Etpp: end time of the termination phase, IP: induction period, $k_{\mathrm{f}}$ : rate constant of 579 ROOHs formation at the propagation phase, $k_{\mathrm{d}}$ : rate constant of ROOHs decomposition at the propagation phase, $k_{1}$ : rate constant of the initiation phase, $\mathrm{M}_{\mathrm{R}}$ : maximum rate of ROOHs formation in the propagation phase, $\mathrm{PP}$ : propagation period, $\mathrm{ROOH}$ : $\mathrm{ROOHs}$ concentration, $[\mathrm{ROOH}]_{\mathrm{IP}}$ : ROOHs concentration at IP point, $[\mathrm{ROOH}]_{\max }$ : maximum concentration of produced ROOHs, $[\mathrm{ROOH}]_{\mathrm{M}_{\mathrm{R}}}$ : ROOHs concentration at the point of the maximum rate of ROOHs formation (or turning point), $t_{M_{R}}$ : occurrence time of maximum rate of ROOHs formation (or turning point).

Figure 2. (a): Sigmoidal curve of hydroperoxides (ROOHs) accumulation in the peroxidation of stripped sunflower oil (Control) containing lecithin (LEC), ferulic acid (FRA), ethyl ferulate (EFR), $\gamma$-oryzanol (GOR) and their combinations at $60{ }^{\circ} \mathrm{C},(\mathrm{b})$ : comparison of graphs of ROOHs production in the presence or absence of lecithin (c): displaying variations in the kinetic parameters of effectiveness $(E)$ and the ratio of oxidation rate $\left(R_{\mathrm{or}}\right)$ of antioxidants in the presence and absence of lecithin, and (d): the relationship between the effectiveness parameter of antioxidants and water content at the IP point.

Figure 3. (a): TEM image of reverse micelles structures in the presence of $\gamma$-oryzanol during the induction period, (b): Schematic figure of the cross-section of a reverse micelle produced during the oxidation process and a display of the dynamics of oxidation products as well as partitioning of the antioxidants under study in this structure (EFR: ethyl ferulate, FRA: Ferulic acid, GOR: $\gamma$-oryzanol, $\mathrm{R}^{*}$ : alkyl radical, RO`: alkoxyl radical, ROO`: peroxyl radical, ROOH: hydroperoxide, WM: water molecule), (c): the changes in the trend of reverse micelles size during peroxidation of stripped sunflower oil at $60{ }^{\circ} \mathrm{C}$. 
600 Figure 4. Relationships between various kinetic parameters in the peroxidation of stripped 601 sunflower oil containing lecithin, ferulic acid, ethyl ferulate, $\gamma$-oryzanol and their combinations

602 at $60{ }^{\circ} \mathrm{C}$. $A$ : antioxidant activity, Etpp: end time of the termination phase, $k_{\mathrm{f}}$ : rate constant of 603 hydroperoxides (ROOHs) formation at the propagation phase, $k_{\mathrm{d}}$ : rate constant of ROOHs 604 decomposition at the propagation phase, $k_{1}$ : rate constant of the initiation phase, $\mathrm{M}_{\mathrm{R}}$ : maximum 605 rate of ROOHs formation in the propagation phase, $[\mathrm{ROOH}]_{\mathrm{IP}}$ : ROOHs concentration at IP 606 point, $[\mathrm{ROOH}]_{\mathrm{M}_{\mathrm{R}}}$ : ROOHs concentration at the point of the maximum rate of ROOHs 607 formation (or turning point), $t_{M_{R}}$ : occurrence time of the maximum rate of ROOHs formation 608 (or turning point), water content IP: water content at the IP point, water content PP: water content 609 at the PP point.

610 Scheme 1. A proposed inhibitory mechanism of ferulic acid and its derivatives, as well as 611 electron resonance delocalization during the oxidation process (as represented by the blue 612 color), and the collision between two antioxidant radicals. 


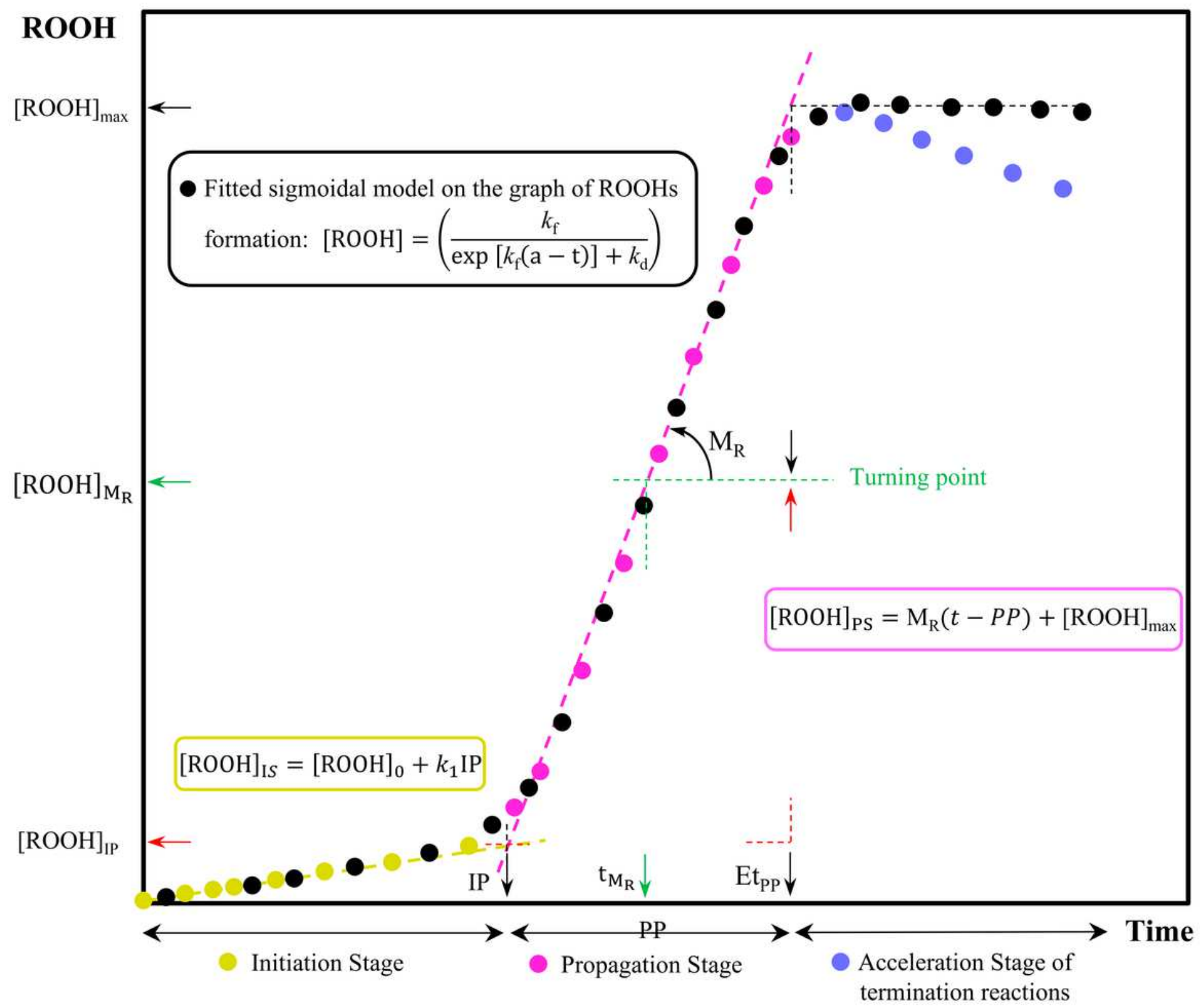

Figure 1

Schematic curve of hydroperoxides (ROOHs) production and a guide of calculated kinetic points. EtPP: end time of the termination phase, IP: induction period, kf: rate constant of ROOHs formation at the propagation phase, $\mathrm{kd}$ : rate constant of $\mathrm{ROOHs}$ decomposition at the propagation phase, $\mathrm{k} 1$ : rate constant of the initiation phase, MR: maximum rate of $\mathrm{ROOHs}$ formation in the propagation phase, PP: propagation period, $\mathrm{ROOH}$ : ROOHs concentration, [ROOH]IP: ROOHs concentration at IP point, $[\mathrm{ROOH}]$ max: maximum concentration of produced $\mathrm{ROOHs}$, [ROOH]_(M_R ): ROOHs concentration at the point of the maximum rate of ROOHs formation (or turning point), $t$ _(M_R ): occurrence time of maximum rate of $\mathrm{ROOHs}$ formation (or turning point). 


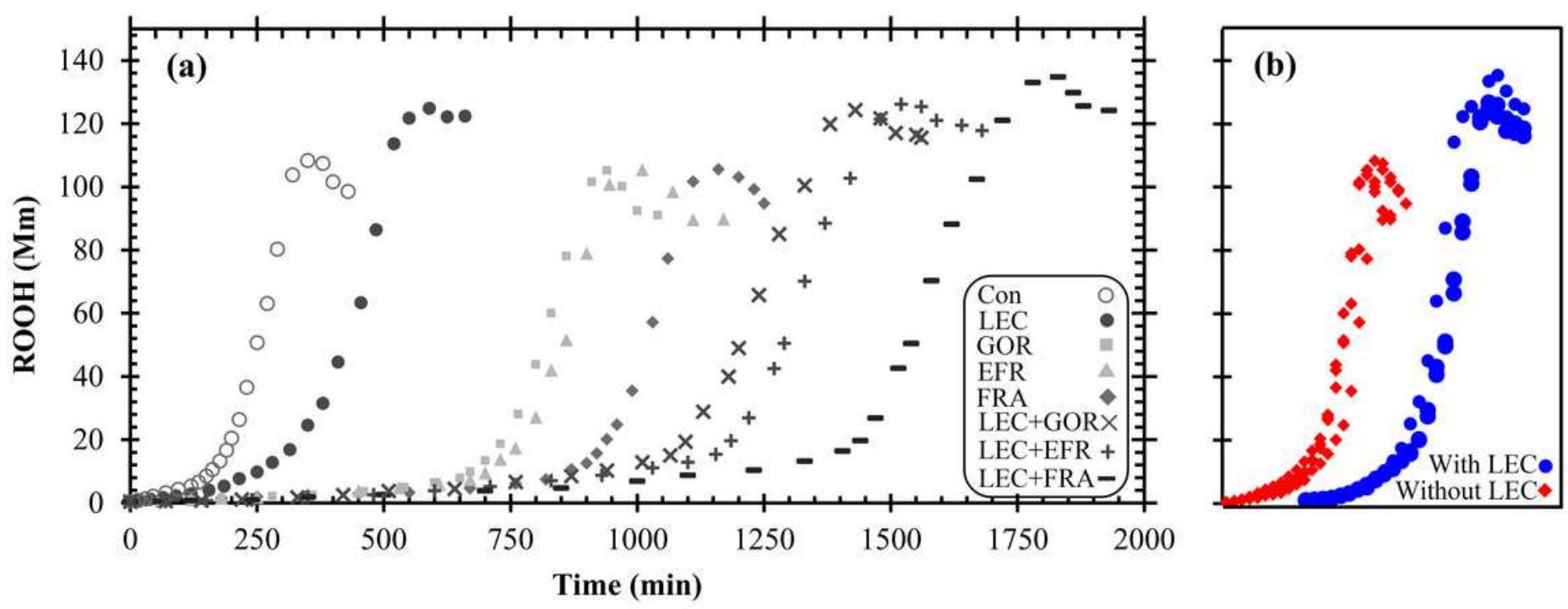

(c)

(d)

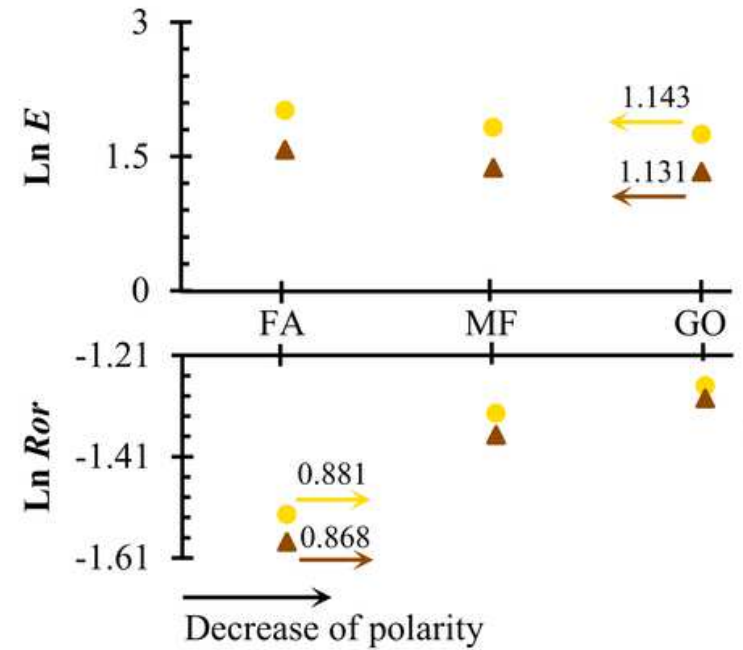

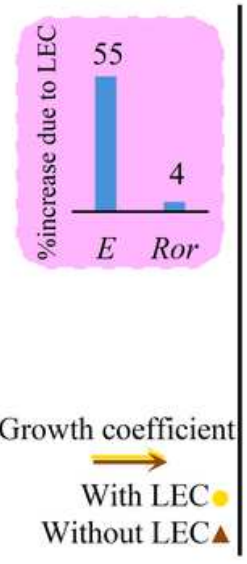

With LEC
Without LEC

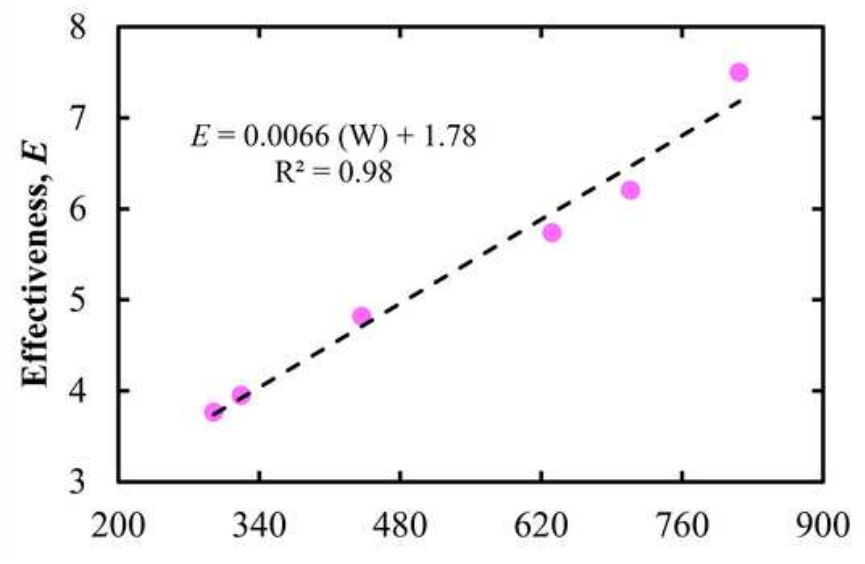

Water content ${ }_{\text {IP }}\left(\mu \mathrm{g} \mathrm{g}^{-1}\right)$

Figure 2

(a): Sigmoidal curve of hydroperoxides (ROOHs) accumulation in the peroxidation of stripped sunflower oil (Control) containing lecithin (LEC), ferulic acid (FRA), ethyl ferulate (EFR), $y$-oryzanol (GOR) and their combinations at $60^{\circ} \mathrm{C},(\mathrm{b})$ : comparison of graphs of $\mathrm{ROOHs}$ production in the presence or absence of lecithin (c): displaying variations in the kinetic parameters of effectiveness $(E)$ and the ratio of oxidation rate (Ror) of antioxidants in the presence and absence of lecithin, and (d): the relationship between the effectiveness parameter of antioxidants and water content at the IP point. 
(a)

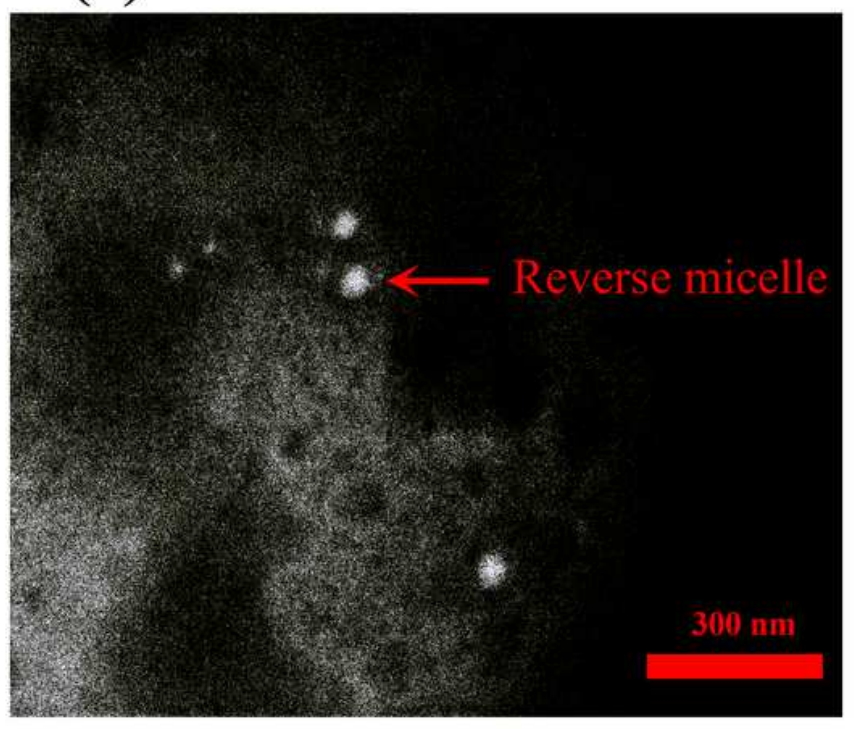

(b)

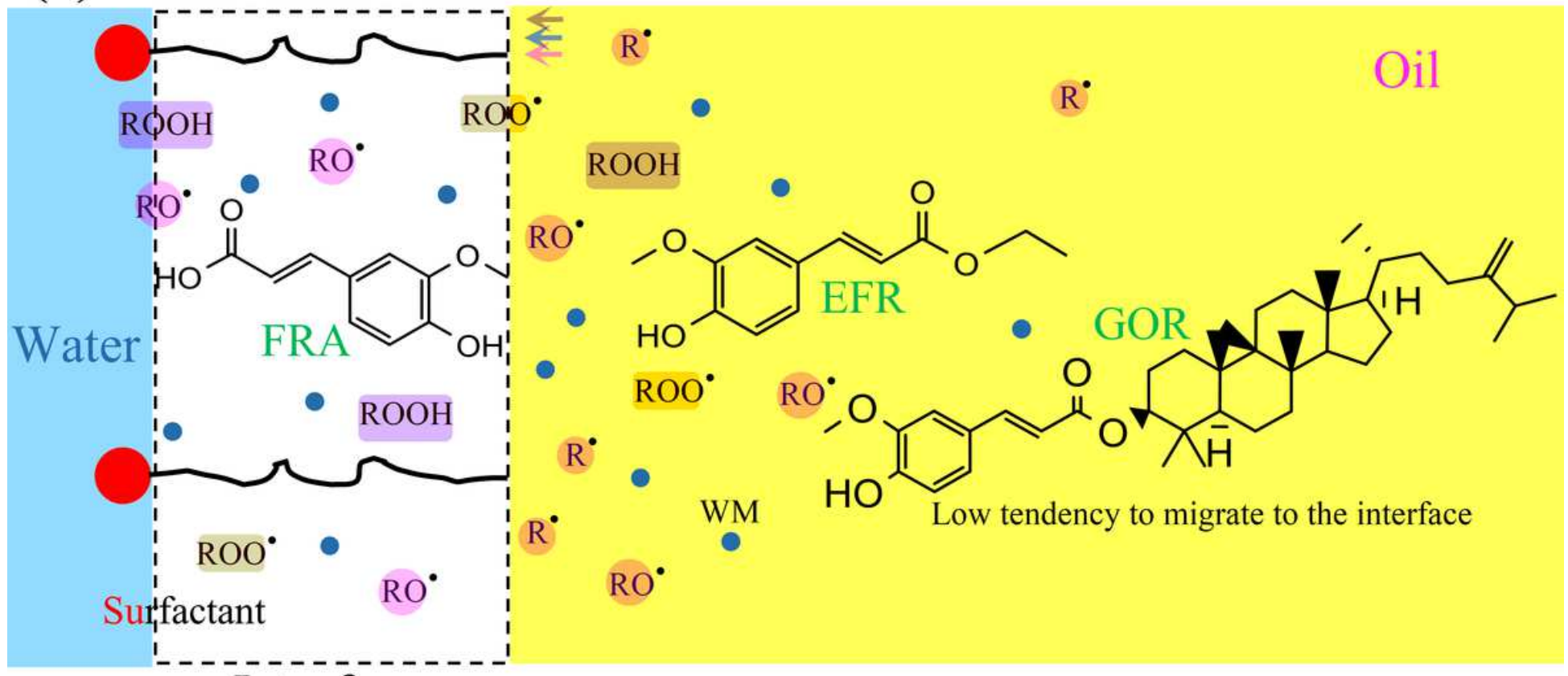

(c)

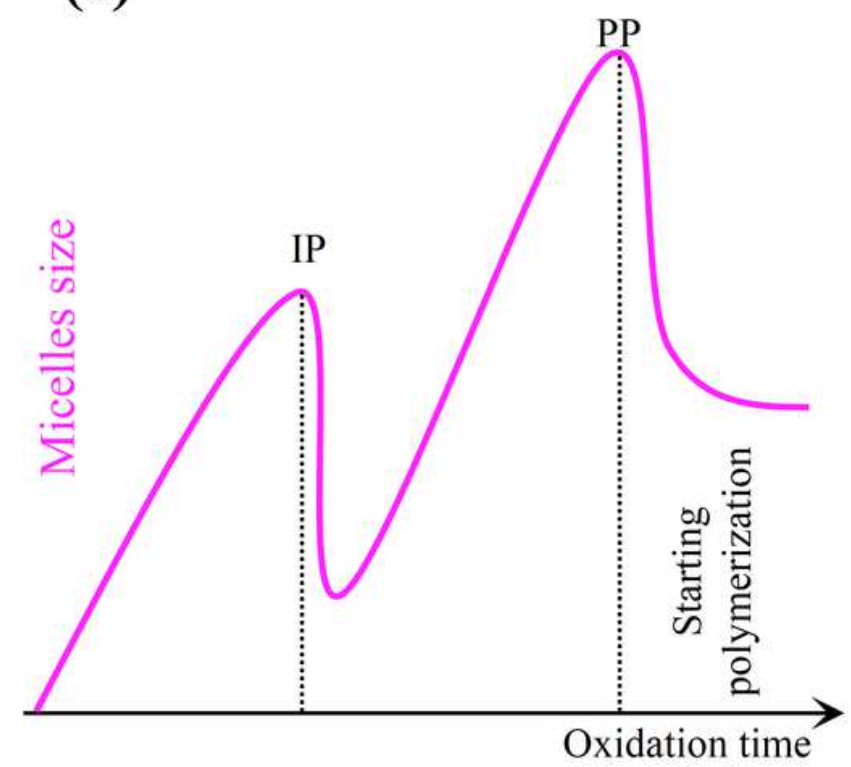

Interface

\section{Figure 3}

(a): TEM image of reverse micelles structures in the presence of $Y$-oryzanol during the induction period,

(b): Schematic figure of the cross-section of a reverse micelle produced during the oxidation process and a display of the dynamics of oxidation products as well as partitioning of the antioxidants under study in this structure (EFR: ethyl ferulate, FRA: Ferulic acid, GOR: $y$-oryzanol, R•: alkyl radical, RO : alkoxyl radical, ROO : peroxyl radical, ROOH: hydroperoxide, WM: water molecule), (c): the changes in the trend of reverse micelles size during peroxidation of stripped sunflower oil at $60^{\circ} \mathrm{C}$. 
(a)

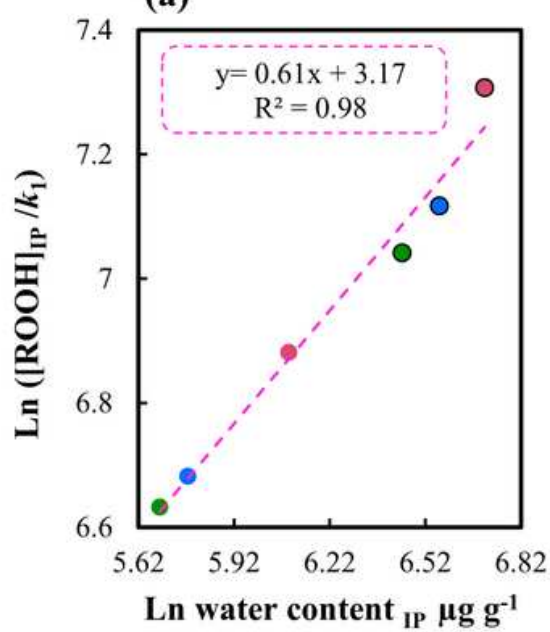

(b)

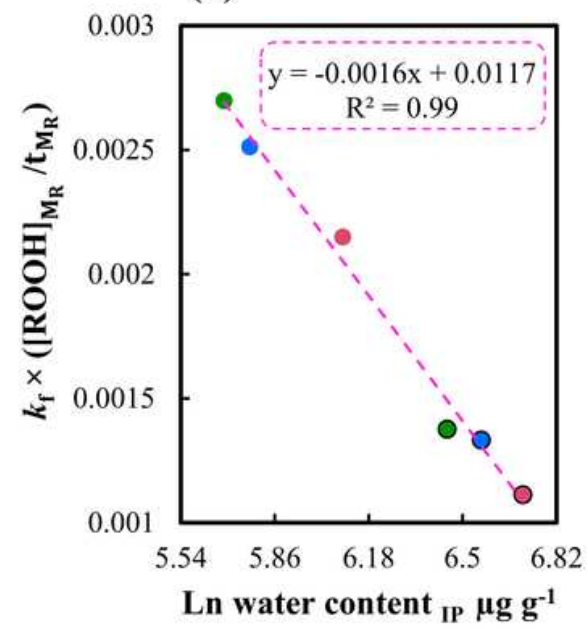

(c)

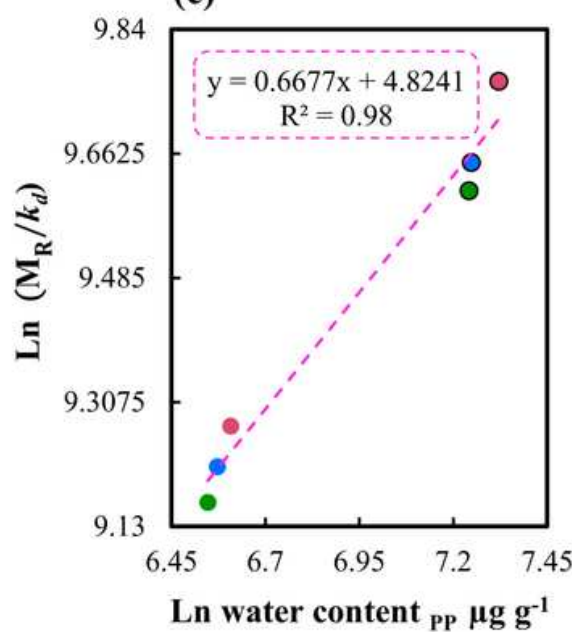

(d)

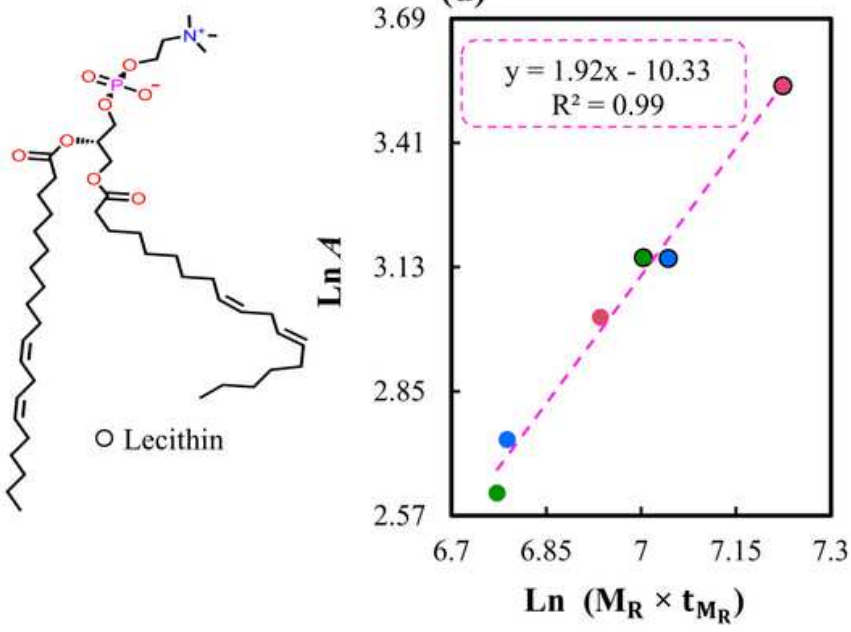

(e)

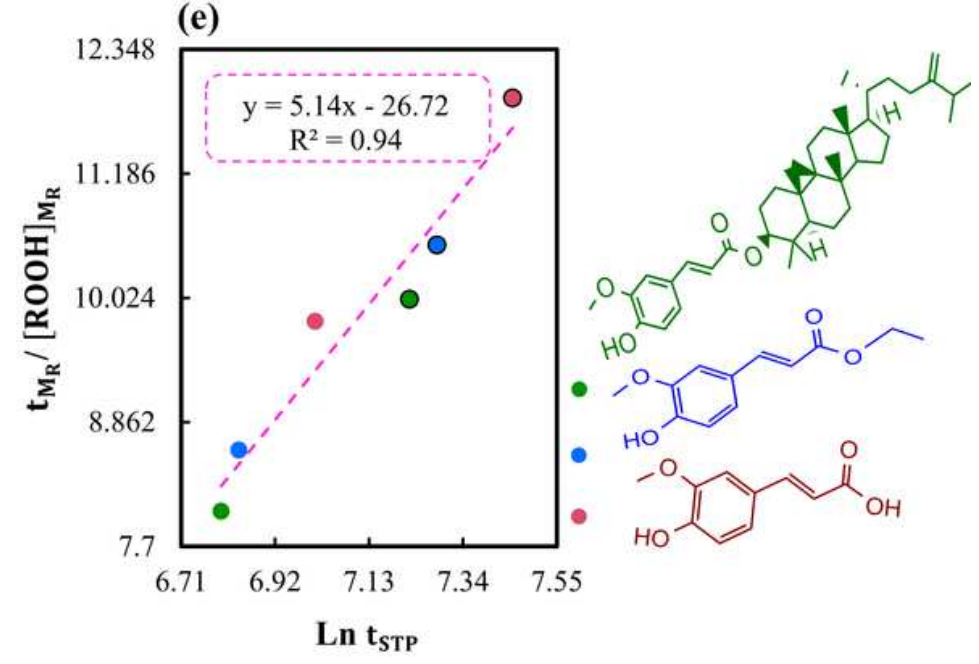

Figure 4

Relationships between various kinetic parameters in the peroxidation of stripped sunflower oil containing lecithin, ferulic acid, ethyl ferulate, $y$-oryzanol and their combinations at $60^{\circ} \mathrm{C}$. A: antioxidant activity, EtPP: end time of the termination phase, kf: rate constant of hydroperoxides (ROOHs) formation at the propagation phase, $\mathrm{kd}$ : rate constant of $\mathrm{ROOHs}$ decomposition at the propagation phase, $\mathrm{k} 1$ : rate constant of the initiation phase, MR: maximum rate of $\mathrm{ROOHs}$ formation in the propagation phase, [ROOH]IP: ROOHs concentration at IP point, [ROOH]_(M_R ): ROOHs concentration at the point of the maximum rate of $\mathrm{ROOHs}$ formation (or turning point), $\mathrm{t}\left(\mathrm{M} \_\mathrm{R}\right)$ : occurrence time of the maximum rate of $\mathrm{ROOHs}$ formation (or turning point), water content IP: water content at the IP point, water content PP: water content at the PP point.

\section{Supplementary Files}

This is a list of supplementary files associated with this preprint. Click to download.

- Graphicalabstract.tif 
- Highlights.docx

- OnlineScheme1.png 